\title{
Electrical impedance tomography clues to detect pulmonary thrombosis in a teenager with COVID-19
}

\author{
Flávia Andrea Krepel Foronda ${ }^{1}$ (1) - Leonardo Rocha Fernandes ${ }^{1} \cdot$ Ana Lucia Capelari Lahoz ${ }^{1} \cdot$ Cintia Johnston $^{2}$. \\ Werther Brunow de Carvalho ${ }^{2}$
}

Received: 17 January 2021 / Revised: 12 June 2021 / Accepted: 25 August 2021 / Published online: 24 September 2021

(C) The Author(s), under exclusive licence to Springer-Verlag GmbH Germany, part of Springer Nature 2021

\begin{abstract}
We report a case of pulmonary thrombosis in a teenager during a hypercoagulable state associated with COVID-19 (coronavirus disease 2019) caused by SARS-CoV-2 (severe acute respiratory syndrome coronavirus 2). A condition rare in children and adolescents, pulmonary thrombosis underdiagnosis likely increases morbidity and mortality. A pulmonary thrombosis diagnosis requires a high level of suspicion and relies on the combination of clinical presentation, D-dimer elevation, and computed tomography (CT) pulmonary angiography or ventilation/perfusion scans, imaging techniques that are difficult to perform. Electrical impedance tomography (EIT) has gained attention, as it provides real-time ventilation distribution analysis. In addition, lung pulsatility images can be obtained through this technique using electrocardiogram gating to filter out ventilation. In this case report, the reduced EIT pulsatility corresponded to the perfusion defect found on the CT scan, information that was obtained at the bedside without radiation or contrast exposure.
\end{abstract}

Keywords Children · Coronavirus disease $2019 \cdot$ Electrical impedance tomography $\cdot$ Lung $\cdot$ Pulmonary thrombosis

\section{Introduction}

Pulmonary thrombosis is relatively rare in the pediatric population when compared to adults, but it is highly recommended to consider this complication since undiagnosed cases potentially increase morbidity and mortality [1]. Very recent data relate SARS-CoV-2 (severe acute respiratory syndrome coronavirus 2 ) infection to a hypercoagulable state, and local thrombi formation may follow the activation of the inflammatory processes

Flávia Andrea Krepel Foronda

flikrepel@ foronda.com.br

1 Pediatric Intensive Care Unit of Instituto da Criança, Hospital das Clínicas, University of São Paulo, Avenida Dr. Enéas de Carvalho Aguiar, 647, CEP 05403000 São Paulo, SP, Brazil

2 Department of Pediatrics of Instituto da Criança, Hospital das Clínicas, University of São Paulo, São Paulo, Brazil during the cytokine storms, causing endothelial damage or dysfunction. In addition, SARS-CoV-2 activates the coagulation pathway by binding to the angiotensinconverting enzyme 2 (ACE-2) receptor [2]. Children with this coronavirus disease 2019 (COVID-19)associated hypercoagulation state are at increased risk for thrombosis [3].

\section{Case report}

We present the case of a previously healthy 15 -year-old girl, weighing $64 \mathrm{~kg}$ with a body mass index of $26.3 \mathrm{~kg} / \mathrm{m}^{2}$, who developed fever, odynophagia and coryza. The initial viral panel investigation was negative, but SARS-CoV-2 was detected subsequently in the nasal swab by a polymerase chain reaction (PCR) test. After 4 days, she presented with sudden dyspnea, low oxygen saturation (92\% with the use of a nonrebreather mask) and asymmetrical lung sounds on auscultation. Blood tests revealed leukocytosis, normal platelet count and an elevated D-dimer level (17079 ng/ml). Based on her 
clinical and laboratory presentation, pulmonary thrombosis was considered. Chest computed tomography (CT) pulmonary angiography, showing a filling defect in the left pulmonary artery, confirmed the diagnosis (Fig. 1). The patient was also monitored with electrical impedance tomography (EIT) (Enlight 1800; Timpel, São Paulo, Brazil) and altered electrocardiogram (ECG)-gated pulsatility in the left lung was detected, in agreement with CT pulmonary angiography (Fig. 2). Additional work-up included a normal transthoracic Echocardiogram (with a left ventricle ejection fraction of $66 \%$ and no coronary abnormalities), an ultrasound of the lower limbs that ruled out deep vein thrombosis and a negative thrombophilia screening. The patient was discharged from the pediatric intensive care unit to the ward 4 days after therapeutic low-molecular-weight heparin was started, without any respiratory support.

\section{Discussion}

Pulmonary thrombosis can be diagnosed based on the combination of a patient's clinical history, D-dimer levels, and ventilation/perfusion (V/Q) scans or $\mathrm{CT}$ pulmonary angiography. Both imaging techniques expose patients to radiation and are costly and time consuming, and $\mathrm{CT}$ pulmonary angiography also includes risks related to contrast injection. These exams are therefore difficult to perform in critically unstable children [4].

EIT is a noninvasive, radiation-free method of measuring the relative change in impedances within regions of a cross-section of the thorax and has recently gained attention in patients with respiratory failure as it provides realtime ventilation images $[5,6]$. EIT also produces pulmonary perfusion images, which have not yet been validated in children because they require intravenous injection of hypertonic saline, which acts as a contrast agent [7]. Conversely, ECG gating can filter out ventilation and provide EIT lung pulsatility images without exposure to hypertonic saline. The thoracic electrical impedance is influenced by changes in gas, tissue and blood. The isolation of the EIT signal within the cardiac frequency domain allows determination of pulsatility [8]. Thus, EIT has the advantage of providing continuous monitoring of ventilation and pulsatility at the same time. In some clinical
Fig. 1 Computed tomography (CT) pulmonary angiography in a 15-year-old girl. a, b Coronal (a) and axial (b) images show a central filling defect in the left pulmonary artery, consistent with an occlusive thrombus (CT dose index: $7.46 \mathrm{mGy})$. The arrows indicate the thrombus
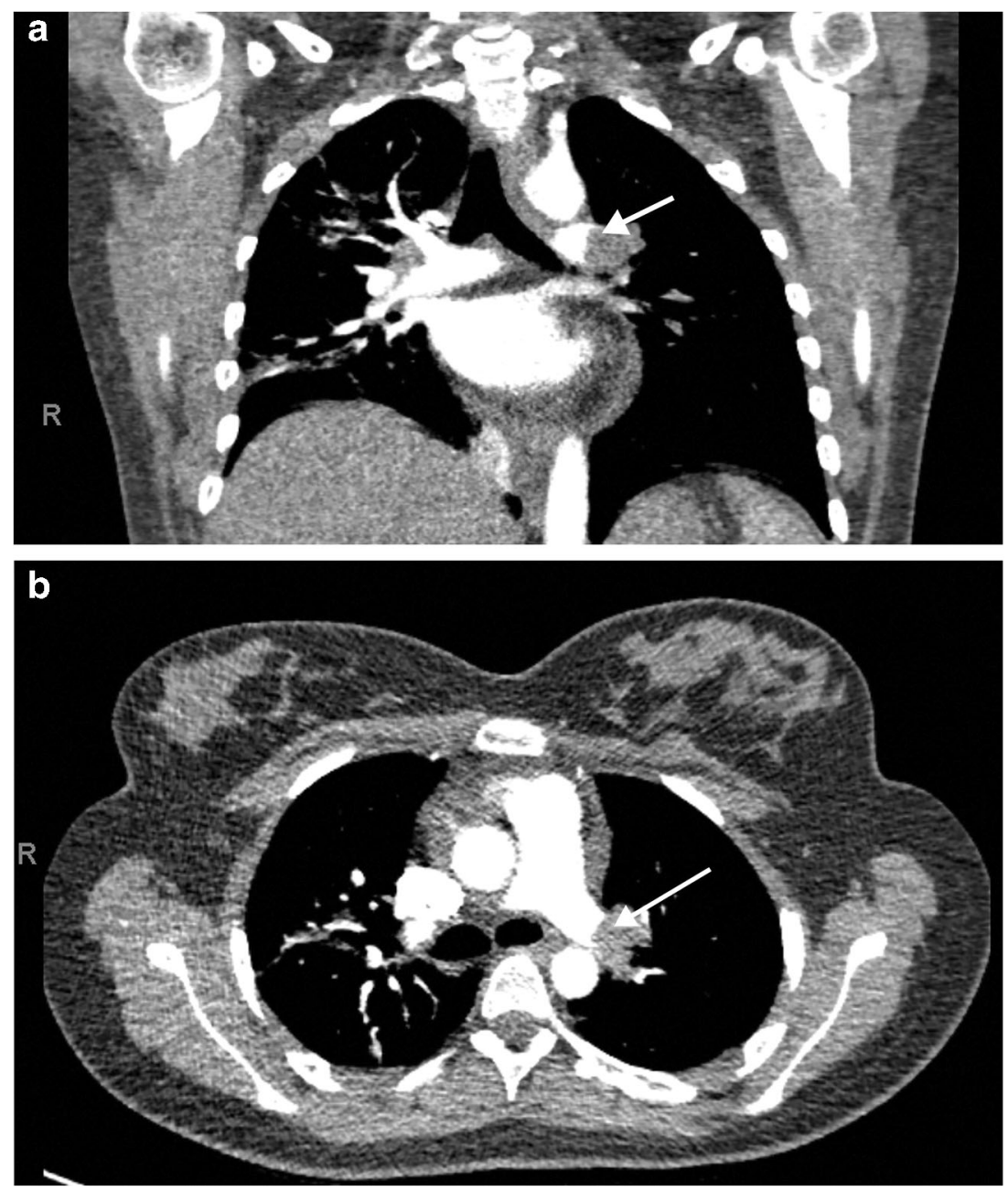


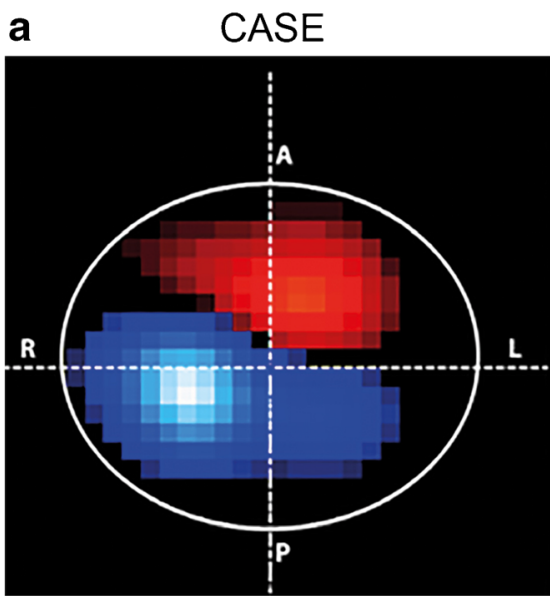

Fig. 2 Axial electrical impedance tomography (EIT) pulsatility maps. The pulsatility images are electrocardiogram gated and filter out the ventilation component in the thoracic impedance variation, thus representing impedance variations related to the cardiac cycle. $\mathbf{a}, \mathbf{b}$ The off-line image shows the heart in red and the pulsatility in the lungs in blue. Of note, the higher detected pulsatility in this EIT algorithm is displayed in white and light blue colors. An image from the 15-year-old

scenarios, mismatch between EIT pulsatility and ventilation maps can suggest pulmonary thrombosis. In our case, patient position affected ventilation distribution, but the pulsatility defect remained unaltered. This finding led to increased suspicion of pulmonary thrombosis. Due to the low EIT spatial resolution, however, a CT angiogram was performed to rule out pulmonary thrombosis [8]. A filling defect was later shown to be responsible for the changes in pulmonary pulsatility (Fig. 3).
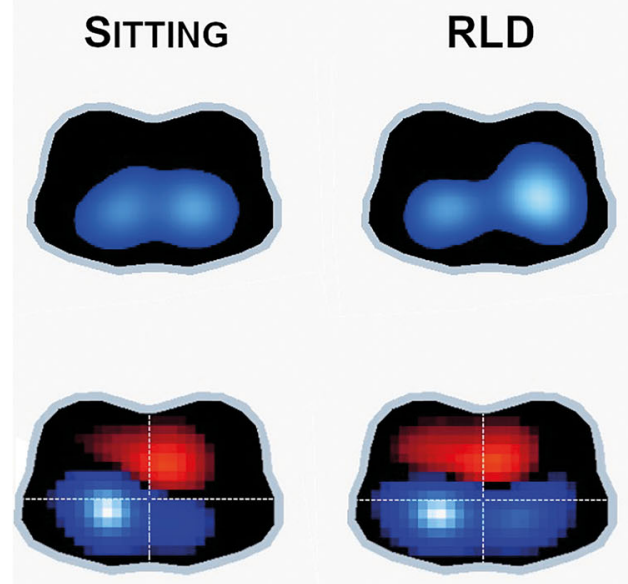

Fig. 3 Axial electrical impedance tomography (EIT) imaging performed in four different positions: sitting, right lateral decubitus $(R L D)$, supine and left lateral decubitus $(L L D)$. The upper figures display EIT lung ventilation maps in scales of blue (the higher the tidal volume detected, the lighter the colors). Noticeably, ventilation distribution varies as the

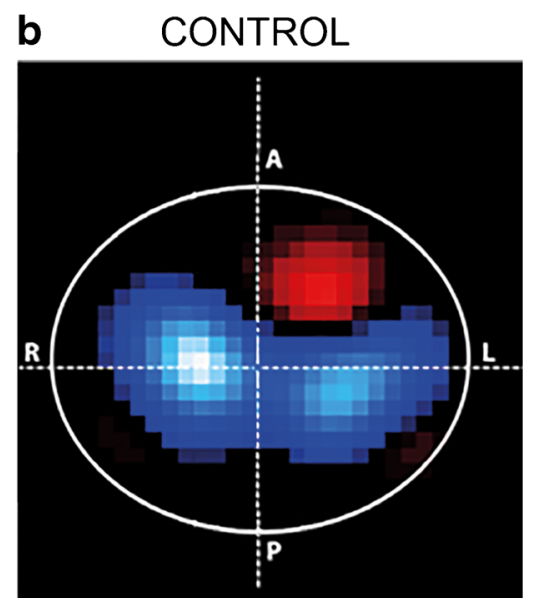

girl (a) shows reduced pulsatility in the left lung, secondary to the pulmonary thrombosis. The dashed lines intersect at the center of the thorax. For comparison, we present an image obtained from another 15year-old girl without ventilation or perfusion abnormalities (b). Note that the pulsatility in the right image is more evenly distributed between the right and left lungs. $A$ anterior, $L$ left, $P$ posterior, $R$ right

\section{Conclusion}

We presented an abnormal pulsatility EIT image secondary to pulmonary thrombosis detected in a teenager with COVID-19 in accordance with CT pulmonary angiography findings. No other risk factors were found, which highlights that a high level of suspicion is warranted in children infected with SARS-CoV-2, since pulmonary thrombosis often remains under-recognized. New diagnostic technologies, such as EIT,

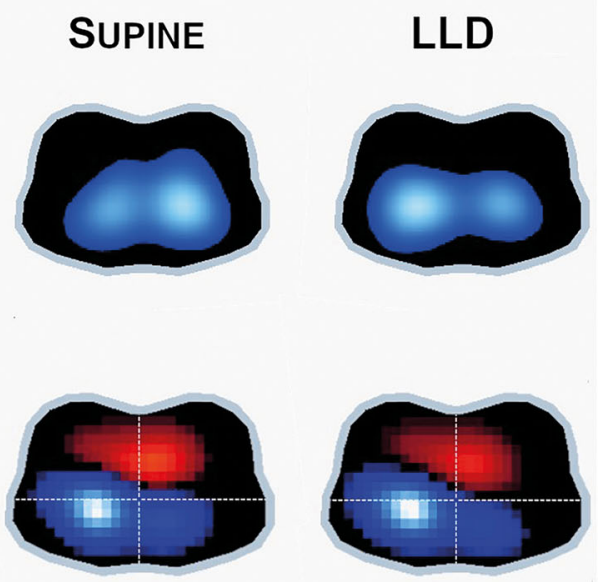

patient changes position. In the lower images, EIT electrocardiogramgated pulsatility maps were not impacted by the four different studied positions. The pulsatility, also presented on a scale of blue to white, is always diminished in the left lung area. The heart position is in red 
might help in the diagnosis and treatment monitoring of respiratory symptoms in critically ill children.

Supplementary Information The online version contains supplementary material available at https://doi.org/10.1007/s00247-021-05199-1.

Acknowledgments We thank Dr. Lisa Suzuki for the valuable contribution in the interpretation of the CT pulmonary angiography.

\section{Declarations}

Conflicts of interest None

\section{References}

1. Zaidi AU, Hutchins KK, Rajpurkar M (2017) Pulmonary embolism in children. Front Pediatr 5:170
2. Connors JM, Levy JH (2020) Thromboinflammation and the hypercoagulability of COVID-19. J Thromb Haemost 18:1559-1561

3. Mitchell WB (2020) Thromboinflammation in COVID-19 acute lung injury. Paediatr Respir Rev 35:20-24

4. Kearon C, de Wit K, Parpia S et al (2019) Diagnosis of pulmonary embolism with D-dimer adjusted to clinical probability. N Engl J Med 381:2125-2134

5. Davies P, Yasin S, Gates S et al (2019) Clinical scenarios of the application of electrical impedance tomography in paediatric intensive care. Sci Rep 9:5362

6. Durlak W, Kwinta P (2013) Role of electrical impedance tomography in clinical practice in pediatric respiratory medicine. ISRN Pediatr 2013:529038

7. Nguyen DT, Jin C, Thiagalingam A, McEwan AL (2012) A review on electrical impedance tomography for pulmonary perfusion imaging. Physiol Meas 33:695-706

8. Carlisle HR, Armstrong RK, Davis PG et al (2010) Regional distribution of blood volume within the preterm infant thorax during synchronised mechanical ventilation. Intensive Care Med 36:21012108

Publisher's note Springer Nature remains neutral with regard to jurisdictional claims in published maps and institutional affiliations. 